\title{
Maintenance of the latent reservoir by pyroptosis and superinfection in a fractional order HIV transmission model
}

\author{
Ana R.M. Carvalho ${ }^{a}$ (D), Carla M.A. Pinto ${ }^{*} b$ (D) and João Nuno Tavares ${ }^{c}$ (D) \\ ${ }^{a}$ Faculty of Sciences, University of Porto, Rua do Campo Alegre s/n, 4440-452 Porto, Portugal \\ ${ }^{b}$ School of Engineering, Polytechnic of Porto, Center for Mathematics of the University of Porto, Rua Dr \\ António Bernardino de Almeida 431, 4249-015 Porto, Portugal \\ ${ }^{c}$ Faculty of Sciences, University of Porto, Rua do Campo Alegre s/n, 4440-452 Porto, Portugal \\ up200802541@fc.up.pt, cap@isep.ipp.pt,jntavar@fc.up.pt
}

\section{ARTICLE INFO}

Article History:

Received 25 July 2018

Accepted 19 November 2018

Available 27 July 2019

Keywords:

Latent reservoir

Pyropstosis

Superinfection

$H I V$

Fractional model

AMS Classification 2010:

26A33; 92D30; $34 A 08 ; 34 K 20$

\section{ABSTRACT}

We focus on the importance of pyroptosis and superinfection on the maintenance of the human immunodeficiency virus (HIV) latent reservoir on infected patients. The latent reservoir has been found to be crucial to the persistence of low levels of viral loads found in HIV-infected patients, after many years of successfully suppressive anti-retroviral therapy (ART). This reservoir seems to act as an archive for strains of HIV no longer dominant in the blood, such as wild-type virus. When a patient decides to quit therapy there is a rapid turnover and the wild-type virus re-emerges. Thus, it is extremely important to understand the mechanisms behind the maintenance of this reservoir. For that, we propose a fractional order model for the dynamics of HIV, where pyroptosis and superinfection are considered. The model is simulated for biological meaningful parameters and interesting patterns are found. Our results are interpreted for clinical appreciation.

\section{Introduction}

HIV is associated with impairment and destruction of the immune system's response, mostly by depletion of $\mathrm{CD}^{+}{ }^{+} \mathrm{T}$ cells. HIV infects several types of these cells, but its primary targets are the $\mathrm{CD} 4^{+} \mathrm{T}$ helper cells. The depletion of these cells may have destructive effects in immune regulation [1. These include reduced antibody development capacity for new attackers, abnormal function of macrophages and decrease in production of chemical messengers.

A fraction of HIV infected $\mathrm{CD} 4^{+} \mathrm{T}$ cells enter a latency state. In this state, the cells do not produce new virus. HIV can remain inside these cells for years, forming reservoirs, which constitute major obstacles for the eradication of HIV. Cells in the latent state escape treatment for HIV. Current anti-retroviral drugs can suppress HIV to undetectable levels, but cannot completely eradicate it 2]. Latently infected cells may be infected by HIV, although with slower kinetics than activated $\mathrm{T}$ cells. Productive superinfection of these latent cells would eliminate virus genome through cell death. A similar effect may be obtained from the induction of pyroptosis of latent cells, in cell-to-cell transmission. Pyroptosis is a process which leads to the destruction of latent $T$ cells, by causing an intensely inflammatory form of programmed cell death, where cytoplasmic contents and pro-inflammatory cytokines are released [3].

Mathematical models have largely been used to predict the dynamics of infections. In 2006, Kim et al 4 study the factors influencing the persistence of the latent reservoir and of low viral load in HIV infected patients, under antiretroviral therapy (ART). They consider that $\mathrm{T}$

*Corresponding Author 
cells can undergo bystander proliferation, without producing active virus, and assume that the latent cells' activation rate decreases with time on ART. The results of the model point to a combined contribution of intrinsic physiological patients' parameters, such as the minimum activation rate or the net regeneration rate of latently infected cells, to explain the persistence of the latent reservoir and of low viral loads. In 2009, Rong et al [5] review several mathematical models for HIV dynamics proposed in the literature. They focus on the quantitative events underlying HIV latency, on the reservoir stability, on the low-level viremia persistence and on the emergence of intermittent viral blips. The authors also distinguish treatment options for each case. In 2015, Wang et al [6] develop a mathematical model to study the pyroptosis mechanism, a programmed cell death, and show how pyroptosis explains the slow time scale of $\mathrm{CD}^{+}{ }^{+} \mathrm{T}$ cells depletion and its contribution to the persistence of latently infected cells. Conway et al [7] describe a mathematical model for the dynamics of HIV to capture the interactions between target cells, productively infected cells, latently infected cells, virus, and cytotoxic T lymphocytes (CTLs). The model provides a CTL response interval for which patients either present viral rebound or post-treatment control, depending on the size of the latent reservoir when treatment finishes. Outside this interval, for lower values, the patients always rebound and for higher values the patients behave as elite controllers. In 2017, Wodarz et al [8] use mathematical models to explain the fundamental mechanisms of the size and of the composition of the latent reservoir in HIV infection. The analysis of the model suggests that though pyroptosis/superinfection are significant factors that influence the dynamics of latency, additional mechanisms might also play a significant role. In particular, abortative infections, higher activation status of cells due to high virus load, the carrying capacity of the latent reservoir.

\subsection{Fractional calculus}

Many mathematical models have a close proximity to reality, however, they are not able to describe it perfectly. Therefore, there is a need to build more accurate models, with the aim of providing better fittings to real data. As such, the Fractional Calculus is one of the most precise tools to refine the description of a series of phenomena present in the most diverse areas of knowledge, namely in engineering, physics, biology, and others 9 -14].
There are several and important definitions for a fractional order derivative. The most well-studied are the Riemann-Liouville (RL), the GrünwaldLetnikov (GL), and the Caputo formula (C). We consider the interval $(0, t)$ instead of $(a, t)$, for simplification. Now, let $y(\tau)$ be a smooth function in every interval $(0, t), t \leq T$. The RL definition reads:

$$
D_{R L}^{\alpha} y(t)= \begin{cases}\frac{1}{\Gamma(m-\alpha)} \frac{d^{m}}{d t^{m}} \int_{0}^{t} \frac{y(\tau)}{(t-\tau)^{\alpha+1-m}}, & m-1 \leq \alpha<m \\ \frac{d^{m} y(t)}{d t^{m}}, & \alpha=m\end{cases}
$$

The Caputo definition is written as:

$$
D_{C}^{\alpha} y(t)= \begin{cases}\frac{1}{\Gamma(m-\alpha)} \int_{0}^{t} \frac{y^{m}(\tau)}{(t-\tau)^{\alpha+1-m}}, & m-1 \leq \alpha<m \\ \frac{d^{m} y(t)}{d t^{m}}, & \alpha=m\end{cases}
$$

The GL definition is equivalent to the RL formula and is based on finite differences. It is given by:

$$
D_{G L}^{\alpha} y(t)=\lim _{h \rightarrow 0} h^{-\alpha} \sum_{k=0}^{n}(-1)^{k} \frac{\Gamma(\alpha+1)}{k ! \Gamma(\alpha-k+1)} y(x-k h), n h=x .
$$

Diethelm [10] demonstrates that a non-integer order model simulates the dynamics of data from the 2009 outbreak of dengue fever, on the Cape Verde islands, more accurately than an integer first order model. The author also shows that the dynamics of the human and of the mosquito populations are modeled by different orders of the fractional derivative. In 2017, Pinto et al [11] study a fractional order model for HIV infection where the dynamics of the latent $\mathrm{CD} 4^{+} \mathrm{T}$ cells, macrophages and cytotoxic $\mathrm{T}$ lymphocytes (CTLs) are considered. The simulations of the model suggest that the order of the fractional derivative is associated to a decrease in the severity of the disease. Namely, are observed decreased values of infected $\mathrm{CD}^{+} \mathrm{T}$ cells and virus with $\alpha$. Moreover, the results of the simulations of the model for relevant parameters, such as the fraction of uninfected $\mathrm{CD} 4^{+} \mathrm{T}$ cells that become latently infected, and the CTLs proliferation rate due to infected $\mathrm{CD} 4^{+} \mathrm{T}$ cells, are biologically acceptable, for all values of $\alpha$. Arshad et al [13] present a non-integer order mathematical model for HIV infection, to study the degree of $\mathrm{T}$ cell depletion caused by viral cytopathology. The results of the model point to the use of the fractional derivative as a parameter to vary to provide better fits to the data of each HIV infected individual. Each individual has its own specificities which are better captured by a non-integer model. Moreover, these models can help doctors choosing the optimal dosage and verify its effects for each individual. 
With the aforesaid ideas in mind, in this paper, we propose a fractional order model for HIV dynamics, where latency, pyroptosis and superinfection are considered. The model is given in Section 2 Its reproduction number and the stability of the disease-free equilibrium are done in Section 3. In Section 4, we analyze the global stability of the disease free equilibrium, and the sensitivity analysis is done in Section 5. In Section 6, we simulate the model for epidemiologically relevant parameters and discuss the results. Finally in Section 7 . we state the main conclusions of this work.

\section{The model}

The uninfected $\mathrm{CD} 4^{+} \mathrm{T}$ cells, $T(t)$, are produced at rate $s$ and die at rate $d$. These cells proliferate exponentially at a rate $r$, until reaching the carrying capacity $K$. They are infected by HIV or by infected $\mathrm{CD} 4^{+} \mathrm{T}$ cells at rates $\beta$ and $\beta_{1}$, respectively. A fraction, $(1-q)$, of infected $\mathrm{CD} 4^{+}$ $\mathrm{T}$ cells becomes latently infected, $L(t)$, and the other fraction, $q$, is actively infected, $I(t)$. The latently infected $\mathrm{CD} 4^{+} \mathrm{T}$ cells become productively infected at a rate $g$ and die at a rate $a_{L}$. The latently infected cells can be successfully superinfected by productive virus at rate $f q \beta$. As the productive infection rate of latently $\mathrm{CD} 4^{+} \mathrm{T}$ cells is lower than that of infected $\mathrm{CD} 4^{+} \mathrm{T}$ cells, we considered the parameter $f<1$. When infected by $\mathrm{CD}^{+} \mathrm{T}$ cells, the latently $\mathrm{CD} 4^{+} \mathrm{T}$ cells die by pyroptosis, which is a form of cell death. Thus, cell-to-cell transmission contributes for cells' death at rate $\beta_{1}$. The infected $\mathrm{CD} 4^{+} \mathrm{T}$ cells, $I(t)$, die at a rate $a_{I}$. HIV, $V(t)$, is produced by the infected $\mathrm{CD} 4^{+} \mathrm{T}$ cells at a rate $p$ and is cleared at a rate $c$. The nonlinear system of fractional-order differential equations describing the dynamics of the model is given by:

$$
\begin{aligned}
\frac{d^{\alpha} T}{d t^{\alpha}}= & s^{\alpha}-d^{\alpha} T+r^{\alpha} T\left(1-\frac{T}{K}\right)-\beta^{\alpha} T V-\beta_{1}^{\alpha} T I \\
\frac{d^{\alpha} L}{d t^{\alpha}}= & (1-q)\left(\beta^{\alpha} T V+\beta_{1}^{\alpha} T I\right)-a_{L}^{\alpha} L-f q \beta^{\alpha} L V \\
& -\beta_{1}^{\alpha} L I-g^{\alpha} L \\
\frac{d^{\alpha} I}{d t^{\alpha}}= & q\left(\beta^{\alpha} T V+\beta_{1}^{\alpha} T I\right)-a_{I}^{\alpha} I+f q \beta^{\alpha} L V+g^{\alpha} L \\
\frac{d^{\alpha} V}{d t^{\alpha}}= & p^{\alpha} I-c^{\alpha} V
\end{aligned}
$$

where $\alpha \in(0,1]$ is the order of the fractional derivative, and ${ }^{\alpha}$ represents the $\cdot$ to the power of $\alpha$. When $\alpha=1$, then the model is the integer order counterpart. The fractional derivative of the proposed model is used in the Caputo sense.

\section{Reproduction number}

In this section, we compute the reproduction number of model (1), $R_{0}$, and the local stability of its disease-free equilibrium. The basic reproduction number is defined as the number of $\mathrm{CD} 4^{+} \mathrm{T}$ cells which are infected by one single cell entering a completely susceptible population. We begin by computing the reproduction number of system (11), $R_{0}$. We use the next generation method [15]. The disease-free equilibrium of model (11) is given by:

$$
\begin{aligned}
P_{0} & =\left(T_{0}, L_{0}, I_{0}, V_{0}\right) \\
& =\left(\frac{K^{\alpha}\left[r^{\alpha}-d^{\alpha}+\sqrt{\left(r^{\alpha}-d^{\alpha}\right)^{2}+\frac{4 \alpha_{s} \alpha}{K}}\right]}{2 r^{\alpha}}, 0,0,0\right)
\end{aligned}
$$

Using the notation in [15] on system (1), matrices for the new infection terms, $F$, and the other terms, $V$, are given by:

$$
\begin{gathered}
F=\left(\begin{array}{ccc}
0 & (1-q) \beta_{1}^{\alpha} T_{0} & (1-q) \beta^{\alpha} T_{0} \\
0 & q \beta_{1}^{\alpha} T_{0} & q \beta^{\alpha} T_{0} \\
0 & 0 & 0
\end{array}\right) \\
V=\left(\begin{array}{ccc}
g^{\alpha}+a_{L}^{\alpha} & 0 & 0 \\
-g^{\alpha} & a_{I}^{\alpha} & 0 \\
0 & -p^{\alpha} & c^{\alpha}
\end{array}\right)
\end{gathered}
$$

The associative basic reproduction number is written as:

$$
R_{0}=\rho\left(F V^{-1}\right)=\frac{T_{0}\left(p^{\alpha} \beta^{\alpha}+c^{\alpha} \beta_{1}^{\alpha}\right)\left(q a_{L}^{\alpha}+g^{\alpha}\right)}{c^{\alpha} a_{I}^{\alpha}\left(g^{\alpha}+a_{L}^{\alpha}\right)}
$$

where $\rho$ indicates the spectral radius of $F V^{-1}$. The linearization matrix of model (11) around the disease-free equilibrium, $P_{0}$, is:

$$
M_{1}=\left(\begin{array}{cccc}
-\sqrt{\left(r^{\alpha}-d^{\alpha}\right)+\frac{4 r^{\alpha} g^{\alpha}}{K}} & 0 & -\beta_{1}^{\alpha} T_{0} & -\beta^{\alpha} T_{0} \\
0 & -a_{L}^{\alpha}-g^{\alpha} & (1-q) \beta_{1}^{\alpha} T_{0} & (1-q) \beta^{\alpha} T_{0} \\
0 & g^{\alpha} & q \beta_{1}^{\alpha} T_{0}-a_{I}^{\alpha} & q \beta^{\alpha} T_{0} \\
0 & 0 & p^{\alpha} & -c^{\alpha}
\end{array}\right)
$$

Stability of $P_{0}$ can be determined using the following lemmas:

Lemma 1. (Theorem 2, [16])

Let $\alpha\left(=\frac{p}{q}\right)$ where $p, q \in \mathrm{Z}^{+}$and $g d c(p, q)=1$. Define $M=q$, then the disease-free equilibrium $P_{0}$ of the system (1) is asymptotically stable if $|\arg (\lambda)|>\frac{\pi}{2 M}$ for all roots $\lambda$ of the following equation

$$
\operatorname{det}\left(\operatorname{diag}\left[\lambda^{M \alpha} \lambda^{M \alpha} \lambda^{M \alpha} \lambda^{M \alpha}\right]-M_{1}\right)=0
$$

Lemma 2. The disease-free equilibrium $P_{0}$ of the system (1) is unstable if $R_{0}<1$. 
Proof. Expanding,

$$
\operatorname{det}\left(\operatorname{diag}\left[\lambda^{M \alpha} \lambda^{M \alpha} \lambda^{M \alpha} \lambda^{M \alpha}\right]-M_{1}\right)=0
$$

we have the following equation in terms of $\lambda$ :

$$
\begin{aligned}
& {\left[\lambda^{M \alpha}+\sqrt{\left(r^{\alpha}-d^{\alpha}\right)+\frac{4 r^{\alpha} s^{\alpha}}{K}}\right]\left[\lambda^{3 M \alpha}+\left(a_{L}^{\alpha}+g^{\alpha}+a_{I}^{\alpha}+c^{\alpha}-q \beta_{1}^{\alpha} T_{0}\right) \lambda^{2 M \alpha}\right.} \\
& +\left(c^{\alpha}\left(a_{L}^{\alpha}+g^{\alpha}+a_{I}^{\alpha}\right)+\left(a_{L}^{\alpha}+g^{\alpha}\right) a_{I}^{\alpha}-T_{0}\left(\beta_{1}^{\alpha}\left(q c^{\alpha}+q a_{L}^{\alpha}+g^{\alpha}\right)+\beta^{\alpha} q p^{\alpha}\right)\right) \lambda^{M \alpha} \\
& \left.+\left(a_{L}^{\alpha}+g^{\alpha}\right) a_{I}^{\alpha} c^{\alpha}\left(1-R_{0}\right)\right]=0
\end{aligned}
$$

Now arguments of the roots of the equation, $\lambda^{M \alpha}+\sqrt{\left(r^{\alpha}-d^{\alpha}\right)+\frac{4 r^{\alpha} s^{\alpha}}{K}}=0$, are given by:

$$
\arg \left(\lambda_{k}\right)=\frac{\pi}{M \alpha}+k \frac{2 \pi}{M \alpha}>\frac{\pi}{M}>\frac{\pi}{2 M}
$$

where $k=0,1, . .,(M \alpha-1)$.

Thus, using Lemma 1, we show that the diseasefree equilibrium, $P_{0}$, of system (11) is stable if all roots of the polynomial:

$$
\begin{aligned}
& \lambda^{3 M \alpha}+\left(a_{L}^{\alpha}+g^{\alpha}+a_{I}^{\alpha}+c^{\alpha}-q \beta_{1}^{\alpha} T_{0}\right) \lambda^{2 M \alpha}\left(c^{\alpha}\left(a_{L}^{\alpha}+g^{\alpha}+a_{I}^{\alpha}\right)+\left(a_{L}^{\alpha}+g^{\alpha}\right) a_{I}^{\alpha}\right. \\
& \left.-T_{0}\left(\beta_{1}^{\alpha}\left(q c^{\alpha}+q a_{L}^{\alpha}+g^{\alpha}\right)+\beta^{\alpha} q p^{\alpha}\right)\right) \lambda^{M \alpha}+\left(a_{L}^{\alpha}+g^{\alpha}\right) a_{I}^{\alpha} c^{\alpha}\left(1-R_{0}\right)=0
\end{aligned}
$$

have argument greater than $\frac{\pi}{2 M}$, for $R_{0}<1$.

Finally, using Descartes' rule of signs in equation (5), we find that there is exactly one sign change for $R_{0}>1$. Thus there is exactly one positive real root of the aforesaid equation for which the argument is less than $\frac{\pi}{2 M}$. We concluded that, if $R_{0}<1$, the disease-free equilibrium $P_{0}$ of the system (11) is stable.

\section{Global stability of the disease-free equilibria}

In this section, we compute the global stability of the disease-free equilibrium of the model (11). We rewrite model (11) as:

$$
\begin{aligned}
& \frac{d^{\alpha} X}{d t^{\alpha}}=F(X, Z) \\
& \frac{d^{\alpha} Z}{d t^{\alpha}}=G(X, Z), \quad G(X, 0)=0
\end{aligned}
$$

where $X=T$ and $Z=(L, I, V)$, with $X \in \mathbf{R}_{+}$ being the number of uninfected $\mathrm{CD} 4^{+} \mathrm{T}$ cells and $Z \in \mathbf{R}_{+}^{3}$ denoting the number of latent and infected $\mathrm{CD} 4^{+} \mathrm{T}$ cells, and virus.

The disease-free equilibrium is written as $U=\left(X^{\star}, 0\right)$, where $X^{\star}=$ $\left(\frac{K^{\alpha}\left[r^{\alpha}-d^{\alpha}+\sqrt{\left.\left(r^{\alpha}-d^{\alpha}\right)^{2}+\frac{4 r^{\alpha} \alpha^{\alpha}}{K}\right]}\right.}{2 r^{\alpha}}, 0\right)$.

The conditions $\left(H_{1}\right)$ and $\left(H_{2}\right)$ must be met to guarantee the global asymptotic stability of the disease-free equilibrium of the model (1):

$$
\begin{aligned}
\left(H_{1}\right): & \text { For } \frac{d^{\alpha} X}{d t^{\alpha}}=F(X, 0), \\
& X^{\star} \text { is globally asymptotically stable } \\
\left(H_{2}\right): & G(X, Z)=A Z-\hat{G}(X, Z), \hat{G} \geq 0 \\
& \text { for }(X, Z) \in \Upsilon_{1}
\end{aligned}
$$

where $A=D_{Z} G\left(X^{\star}, 0\right)$ is a M-matrix (the offdiagonal elements of $A$ are non-negative) and $\Upsilon_{1}$ is the region where the model makes biological sense. If the system (6) satisfies the conditions in (7) the following theorem holds.

Theorem 1. The fixed point $U=\left(X^{\star}, 0\right)$ is a globally asymptotically stable equilibrium of the system (6) provided that $R_{0}<1$ and that the assumptions in (7) are satisfied.

\section{Proof. Let}

$$
F(X, 0)=\left(s^{\alpha}-d^{\alpha} T+r^{\alpha} T\left(1-\frac{T}{K}\right)\right)
$$

and

$$
A=\left(\begin{array}{ccc}
-g^{\alpha}-a_{L}^{\alpha} & (1-q) \beta_{1}^{\alpha} T_{0} & (1-q) \beta^{\alpha} T_{0} \\
g^{\alpha} & q \beta_{1}^{\alpha} T_{0}-a_{I}^{\alpha} & q \beta^{\alpha} T_{0} \\
0 & p^{\alpha} & -c^{\alpha}
\end{array}\right)
$$

and

$$
\begin{aligned}
& \hat{G}(X, Z)=\left(\begin{array}{c}
\hat{G}_{1}(X, Z) \\
\hat{G}_{2}(X, Z) \\
\hat{G}_{3}(X, Z)
\end{array}\right) \\
& =\left(\begin{array}{c}
(1-q) T_{0}\left(1-\frac{T}{T_{0}}\right)\left(\beta_{1}^{\alpha} I+\beta^{\alpha} V\right)+f q \beta^{\alpha} L V+\beta_{1}^{\alpha} L I \\
q T_{0}\left(1-\frac{T}{T_{0}}\right)\left(\beta_{1}^{\alpha} I+\beta^{\alpha} V\right)-f q \beta^{\alpha} L V \\
0
\end{array}\right)
\end{aligned}
$$

Thus $\hat{G}_{1}(X, Z), \hat{G}_{2}(X, Z) \geq 0$ and $\hat{G}_{3}(X, Z)=$ $0 \Rightarrow \hat{G}(X, Z) \geq 0$. Conditions in (17) are satisfied, thus the disease-free equilibrium of the model (11) is globally asymptotically stable for $R_{0}<1$.

\section{Sensitivity analysis}

In this section we compute the sensitivity indexes of the reproduction number, $R_{0}$ (11). Sensitivity indexes are given in Table 1 and provide information on the variation of the value of $R_{0}$ as a function of each parameter. We follow the procedure proposed in [17]. Generically, when $R_{0}>1$, the epidemics spreads, on the other hand, for $R_{0}<1$ the epidemics halts. 
Table 1. Sensitivity indexes for relevant parameters of model (10).

\begin{tabular}{cc}
\hline Parameter & Sensitivity index sign \\
\hline$\beta$ & + \\
$\beta_{1}$ & + \\
$p$ & + \\
$q$ & + \\
$g$ & + \\
$c$ & - \\
$a_{L}$ & - \\
$a_{I}$ & - \\
\hline
\end{tabular}

\section{Numerical results}

We simulate the model (11) for different values of the order of the fractional derivative, $\alpha$ and for epidemiologically valid parameters. The parameters used in the simulations, based on [7,8], are: $s=10$ day $^{-1}, d=0.015$ day $^{-1}, r=0.03$ day $^{-1}, K=1500 \mathrm{~mm}^{-3}, \beta=0.0001 \mathrm{~mm}^{3}$ day $^{-1}$, $\beta_{1}=0.0001 \mathrm{~mm}^{3}$ day $^{-1}, q=0.95, g=0.001$ day $^{-1}, a_{L}=0.03$ day $^{-1}, f=1 / 7, a_{I}=0.45$ day $^{-1}, p=2000$ day $^{-1}, c=23$ day $^{-1}$, and the initial conditions are: $T(0)=700, L(0)=I(0)=0$ and $V(0)=10$.

Fiures 1 13 depict the number of latently infected cells in the cases of existence and absence of pyroptosis/superinfection. It is observed a higher number of latent cells when there is no pyroptosis/superinfection, for the three values of the order of the fractional derivative, $\alpha$. In both cases, with and without pyroptosis, there is a first increase in the number of latent cells towards a peak and then a convergence to an asymptotic state. Moreover, the behaviour with pyroptosis/superinfection at $\alpha=1$ shows a minimum after the peak and then a rise to the equilibrium state. This may be due to the variation of the HIV viral load, which is related with the phenomenon of pyroptosis/superinfection, as follows. The HIV viral load increase from lower levels is followed by the rise of the latent cells. When the HIV load reaches its peak value, the number of latent cell decreases due to cell death by pyroptosis or by superinfection. As the viral load declines and tends asymptotically to its equilibrium, the latent pool rebounds and increases to some value. Lower viral loads are associated with less pyroptosis and less superinfection, which translates in the perseverance of the latent cells' pool. This rebound feature is rapidly forgotten for smaller values of the order of the fractional derivative $\alpha$, probably due to the memory property, which causes transients to be faster in these systems than in integer order ones.

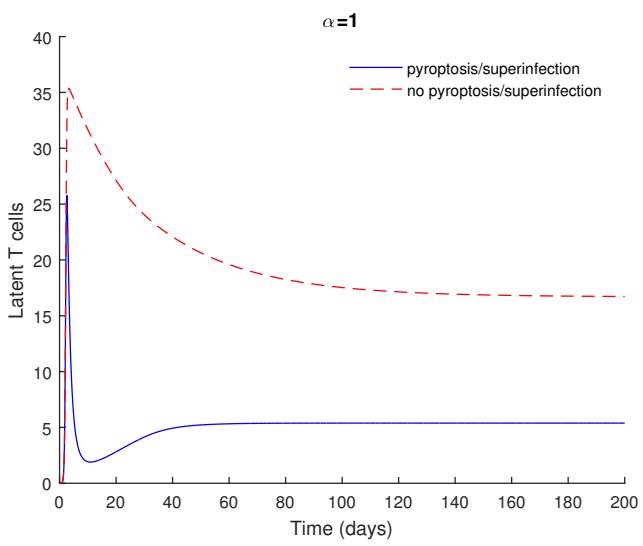

Figure 1. Number of latent cells with and without pyroptosis/superinfection, for $\alpha=1$. Parameter values and initial conditions in the text.

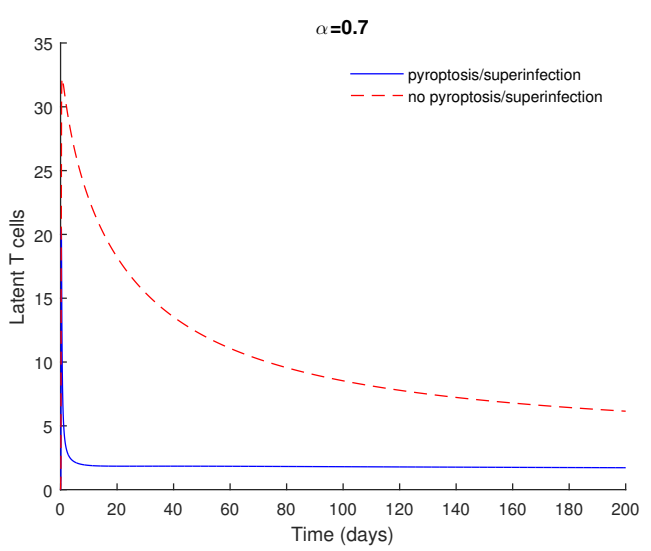

Figure 2. Number of latent cells with and without pyroptosis/superinfection, for $\alpha=0.7$. Parameter values and initial conditions in the text.

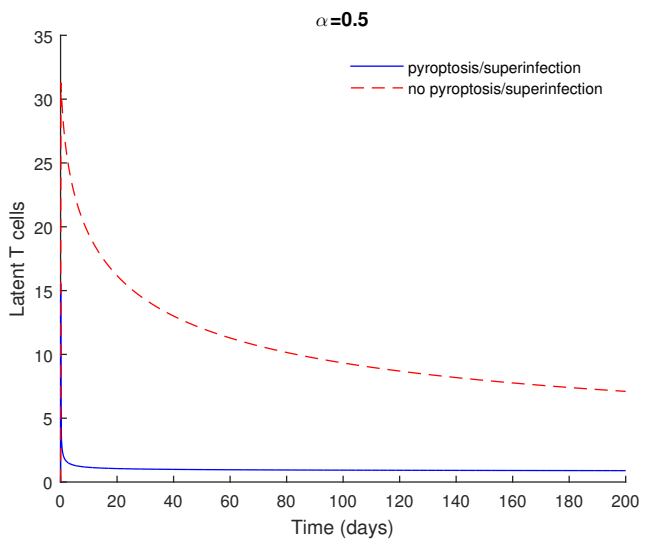

Figure 3. Number of latent cells with and without pyroptosis/superinfection, for $\alpha=0.5$. Parameter values and initial conditions in the text. 


\section{Conclusion}

We proposed a non-integer order mathematical model for HIV infection to study the influence of pyroptosis and superinfection on the maintenance of the latent reservoir. We computed the basic reproduction number and the stability of the disease-free equilibrium. The simulations of the model provide good agreement with experimental data available in the literature concerning the maintenance of the latent reservoir. It is observed that as HIV load increases from lower levels, the latent cells' population also rises. When the viral load reaches its peak, the number of latent cells decreases, due to cell death by pyroptosis and superinfection. As the viral load declines and tends asymptotically to its equilibrium, the latent pool rebounds and increases to some threshold. Thus, pyroptosis and superinfection, are important players in the perseverance of the latent cells' pool in HIV infection.

\section{Acknowledgment}

The authors were partially funded by the European Regional Development Fund through the program COMPETE and by the Portuguese Government through the FCT - Fundação para a Ciência e a Tecnologia under the project PEstC/MAT/UI0144/2013. The research of AC was partially supported by a FCT grant with reference SFRH/BD/96816/2013.

\section{References}

[1] Perelson, A.S., Kirschner, D.E \& De Boer, R. (1993). Dynamic of HIV infection of CD4+ T cells. Mathematical Biosciences, 112, 81-125.

[2] Chavez, L., Calvanese, V \& Verdin, E. (2015). HIV latency is established directly and early in both resting and activated primary CD4 T cells. PLOS Pathogens, 11(6), e1004955.

[3] Doitsh, G., Galloway, N.L.K., Geng, X., Yang, Z., Monroe, K.M., Zepeda, O., Hunt, P.W., Hatano, H., Sowinski, S., Muoz-Arias, I. \& Greene, W.C. (2014). Pyroptosis drives CD4 T-cell depletion in HIV-1 infection. $\mathrm{Na}$ ture, 505(7484), 509-514.

[4] Kim, H. \& Perelson, A.S. (2006). Viral and latent reservoir persistence in HIV-1infected patients on therapy. PLOS Computational Biology, 2(10), e135.

[5] Rong, L. \& Perelson, A.S. (2009). Modeling HIV persistence, the latent reservoir, and viral blips. Journal of Theoretical Biology, 260, 308-331.
[6] Wang, S., Hottz, P., Schechter, M. \& Rong, L. (2015). Modeling the slow CD4+ T cell decline in HIV-infected individuals. PLOS Computational Biology, 11(12), e1004665.

[7] Conway, J.M. \& Perelson, A.S. (2015). Posttreatment control of HIV infection. Proceedings of the National Academy of Sciences, 112(17), 5467-5472.

[8] Wodarz, D., Levy, D.N., Pyroptosis, superinfection, and the maintenance of the latent reservoir in HIV-1 infection. Nature - Scientific Reports, 7(1), 1-10 (2017).

[9] Samko, S., Kilbas, A. \& Marichev, O. (1993). Fractional Integrals and Derivatives: Theory and Applications. London: Gordon and Breach Science Publishers.

[10] Diethelm, K. (2013). A fractional calculus based model for the simulation of an outbreak of dengue fever. Nonlinear Dynamics, 71, 613-619.

[11] Pinto, C.M.A. \& Carvalho, A.R.M. (2017). A latency fractional order model for HIV dynamics. Journal of Computational and Applied Mathematics, 312, 240-256.

[12] Téjado, I., Valério, D., Pérez, E. \& Valério, N. (2017). Fractional calculus in economic growth modelling: the Spanish and Portuguese cases. International Journal of Dynamics and Control, 5(1), 208-222.

[13] Arshad, S., Baleanu, D., Bu, W., Tang, Y., Effects of HIV infection on CD4+ T-cell population based on a fractional-order model. Advances in Difference Equations, 2017(92), 1-14 (2017).

[14] Carvalho, A.R.M., Pinto, C.M.A. \& Baleanu, D. (2018). HIV/HCV coinfection model: a fractional-order perspective for the effect of the HIV viral load. Advances in Difference Equations, 2018(1), 1-22.

[15] Driessche, P. \& Watmough, P. (2002). Reproduction numbers and sub-threshold endemic equilibria for compartmental models of disease transmission. Mathematical Biosciences, $180,29-48$.

[16] Tavazoei, M.S. \& Haeri, M. (2008). Chaotic attractors in incommensurate fractional order systems. Physica D, 237, 2628-2637.

[17] Chitnis, N., Hyman, J.M. \& Cushing, J.M. (2008). Determining important parameters in the spread of malaria through the sensitivity analysis of a mathematical model. Bulletin of Mathematical Biology, 70, 1272-1296. 
Ana R.M. Carvalho is a Doctor in Mathematics since May 2018. Her current research interests comprise the development of mathematical models for epidemiology. She has more than 20 papers published in international journals and conferences. Her h-index is 9 and the $i$-index is 8 .

Carla M.A. Pinto is an Adjunct Professor of the School of Engineering of the Polytechnic of Porto. She is a Doctor in Mathematics since 2004. Her research interests involve: the study of integer-order and fractional order models, using bifurcation theory, stability theory, numerical simulations. Applications: epidemiology, robotics, engineering. She has published original research in several high impact international journals and international conferences. She is Associate Editor of International Journals, has been member of the Scientific Committee and of the International Program Committee of several international conferences, chair of sessions at international conferences. She has more than 799 citations. Her h-index is 15 and the i-index is 20 .

João Nuno Tavares is an Associate Professor at the Faculty of Sciences of the University of Porto (FCUP). He is a Doctor in Physics since 1993. His research interests include recreational mathematics, optimization, computational mathematics, mathematical models in cancer therapy. He is a member of the Scientific Committee of the Department of Mathematics of FCUP since July 2017. He is the Director of the Department of Mathematics since November 2017. He was a member of the Executive Commission of the Department of Mathematics in October 2013, and a member of the Council of Representatives since July 2014. He is the Director of GEMAC - Gabinete de Estatística, Modelação e Aplicações Computacionais. He has organized several international conferences. He publishes papers in international high impact journals.

An International Journal of Optimization and Control: Theories \& Applications (http://ijocta.balikesir.edu.tr)

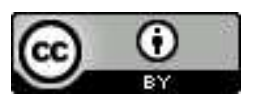

This work is licensed under a Creative Commons Attribution 4.0 International License. The authors retain ownership of the copyright for their article, but they allow anyone to download, reuse, reprint, modify, distribute, and/or copy articles in IJOCTA, so long as the original authors and source are credited. To see the complete license contents, please visit http://creativecommons.org/licenses/by/4.0/. 